\title{
The IncRNA MEC3 promotes trophoblastic cell growth and invasiveness in preeclampsia by acting as a sponge for miR-21, which regulates BMPR2 levels
}

\author{
Huyi Liu, ${ }^{1,2 *}$ Xiangdao Cai, ${ }^{3 *}$ Jia Liu, ${ }^{1}$ Fengxiang Zhang, ${ }^{1}$ Andong He, ${ }^{1}$ Ruiman Li $^{1}$ \\ ${ }^{1}$ Department of Obstetrics and Gynecology, The First Affiliated Hospital of Jinan University, Guangzhou \\ ${ }^{2}$ Department of Obstetrics and Gynecology, Zhongshan People's Hospital Zhongshan Hospital of Sun Yat-sen \\ University, Zhongshan \\ ${ }^{3}$ Department of Orthodontics, Stomatological Clinic, Zhongshan People's Hospital of Sun Yat-sen University, \\ Zhongshan, China \\ *These authors contributed equally to this work.
}

Preeclampsia (PE) is one of the leading causes of maternal morbidity and mortality in pregnant women. This study aimed to investigate the potential impact and regulatory mechanisms of bone morphogenetic protein receptor 2 (BMPR2) on the progression of PE. We obtained placental tissues from pregnant women with PE and normal pregnant women, and the results showed that BMPR2 was expressed at low levels in the tissue from PE women. Genetic knockdown of BMPR2 increased the proliferation and invasion of cultured trophoblast cells, whereas its overexpression reduced these characteristics. Bioinformatics analysis and luciferase reporter gene assays confirmed that BMPR2 is a direct target of miR-21. Overexpression of a miR-21 inhibitor promoted the growth and invasiveness of trophoblast cells, whereas the opposite results were observed for the miR-21 mimic. Furthermore, miR-21 was sponged by the IncRNA MEG3, and shRNA inhibition of MEG3 reduced trophoblast cell growth and invasiveness. miR-21 was upregulated in the tissues from PE women, whereas MEG3 was downregulated, and the two were negatively correlated. Collectively, this study demonstrates that the lncRNA MEG3 acts as a sponge for miR-21, which regulates BMPR2 expression and promotes trophoblast cell proliferation and invasiveness, thereby preventing the development of PE. These findings provide novel insight into a targeted therapy that could be used to treat or prevent the development of PE.

Key words: Preeclampsia; miR-21; lncRNA MEG3; bone morphogenetic protein receptor 2; trophoblast cell.

Correspondence: Ruiman Li, Department of Obstetrics \& Gynecology, The First Affiliated Hospital of Jinan University, Guangzhou, China. E-mail: hqyyck@126.com

Contributions: RL, study concept and experiments design; HL, DC, experiments performing, data analysis, manuscript writing; JL, FZ, results discussion; AH, manuscript revision. All the authors have read and approved the final version of the manuscript and agreed to be accountable for all aspects of the work.

Conflicts of interest: The authors declare conflict of interest.

Ethical approval: Ethical approval was obtained for all experimental procedures from the Ethics Committee from the First Affiliated Hospital of Jinan University, Guangzhou, China.

Patient consent for publication: Written informed consent was obtained from the patients for their anonymized information to be published in this article.

Availability of data and materials: The datasets used and/or analyzed in this study are available from the corresponding author. 


\section{Introduction}

Hypertensive disorders complicating pregnancy (HDCP), pregnancy-specific diseases, include pregnancy-induced hypertension, preeclampsia (PE), and eclampsia. ${ }^{1,2}$ The global incidence of HDCP is about $10 \%$, and HDCP is a leading cause of long-term disability and death in mothers and babies, greatly threatening the life and health of the mother and fetus. ${ }^{3} \mathrm{PE}$ is the most commonly presented form of HDCP, its incidence has increased in recent years, and it often produces serious adverse complications in the mothers and fetuses. ${ }^{4-6}$ To date, the etiology and pathogenesis of $\mathrm{PE}$ are unclear, which directly affects the effectiveness of early diagnosis, prevention, and treatment of PE.

Transforming growth factor- $\square$ (TGF- $\beta$ ) family members include TGF- $\beta$, bone morphogenetic protein (BMP), nodal, and activin, which are widely expressed in different tissues and play roles in cell growth, migration, and apoptosis in various tissues.? BMP receptor 1 (BMPR1) and BMPR2 are downstream receptors for BMPs. BMP, BMPR1, and BMPR2 form a tetrameric complex triggering signal transduction. ${ }^{8}$ In addition, BMPs transmit extracellular signals into cells through BMPRs to stimulate cells to induce a series of physiological changes, thereby regulating cell proliferation, apoptosis, and differentiation. ${ }^{9}$

BMPR2 plays important roles in embryonic development, angiogenesis, and osteogenesis. ${ }^{9,10}$ BMPR2 is composed of 1038 amino acids, with a molecular weight of $115 \mathrm{kDa}$, and is widely expressed in the endothelial and smooth muscle cells of different tissues and organs. ${ }^{11}$ To date, the function of BMPR 2 in HDCP has not been investigated. There is a close correlation between BMPR2 and hypertension. BMPR2 mutations are associated with the development of pulmonary hypertension (PAH), ${ }^{9}$ chronic obstructive pulmonary disease (COPD), ${ }^{12}$ hemorrhagic telangiectasia (HHT), tumors, and obesity. ${ }^{13}$ In pulmonary hypertension, BMPR2 deficiency inhibits the proliferation and differentiation of vascular smooth muscle cells and endothelial cells, and modulation of BMPR2 expression changes the processes of proliferation and apoptosis. ${ }^{9}$

In recent years, noncoding RNAs (ncRNAs) have become of interest in various fields. ncRNAs are divided into long ncRNAs (lncRNAs), microRNAs (miRNAs), and circular RNAs (circRNAs). They control gene transcription and translation through RNA/protein sponging and epigenetic modification, thereby regulating various cellular processes. ${ }^{14-18}$ miRNAs are endogenous single-stranded ncRNAs with a length of 20-22 nucleotides, and they bind to the 3'-untranslated region (3'-UTR) of the target gene to regulate the translation process. ${ }^{19}$ The significance of miRNA involvement in PE development is frequently reported. Studying the role miRNAs play in the placenta may help to identify the pathogenesis and pathological manifestations of placentalrelated diseases, and provide a new scientific basis for prevention and treatment. ${ }^{20}$ Detection of relevant miRNAs in peripheral blood circulation may become an important diagnostic for early screening and diagnosis of PE. miR-21 is highly expressed in PE placental samples, but little is known about its downstream target genes and regulatory mechanisms of action. ${ }^{21}$ lncRNAs are crucial components of transcription and post-transcriptional regulation of gene expression, serving as key nodes for the regulation of transcription activity and mRNA expression of target genes. Some lncRNAs have been implicated in the development of PE, such as the lncRNAs MEG3, TUG1, and MALAT1.22-24 MEG3 is transcribed at low levels in PE samples, and overtranscription of MEG3 promotes trophoblast invasion and metastasis, indicating that $M E G 3$ may prevent the development of $\mathrm{PE}$ and play a critical role in the regulation of PE..$^{22}$ However, its downstream regulatory targets and mechanisms of action are still elusive.
Therefore, the purpose of this study was to explore the relationship between BMPR2 and PE in order to understand the role BMPR2 plays in the development of HDCP. Specifically, we set out to explore the function of BMPR2 in PE, to identify the upstream regulators of BMPR2, and to provide novel insight into the pathogenesis of PE.

\section{Materials and Methods}

\section{Tissue sample collection}

A total of 12 placenta samples were collected from pregnant women with PE who had not had a cesarean section operation (PE group) at the Obstetrics and Gynecology Department of Jinan University Hospital between May 2020 and Feb 2021. An additional 12 placental tissues were obtained from normal pregnant women who delivered by cesarean section without giving birth at full term (control group). Specimens were obtained from the placenta center near the umbilical cord to avoid infarction and thrombosis after the placenta is removed from the uterus. The sample was then washed with sterile phosphate buffered saline and stored in liquid nitrogen. Written consent was obtained from all patients, and the study was approved by the ethics committee from the First Affiliated Hospital of Jinan University.

\section{Cell culture and transfection}

JEG-3 trophoblast cells purchased from the American Type Culture Collection (ATCC, Manassas, VA, USA) were cultured in DMEM (Gibco, Thermo Scientific, Waltham, MA, USA) medium with $10 \%$ FBS (Gibco) and 1\% penicillin-streptomycin (Gibco) at $37^{\circ} \mathrm{C}$ in $5 \% \mathrm{CO}_{2}$, and the medium was replaced with fresh medium every 2 days. When the cell density reached $60-70 \%$, the cells were transfected using Lipofectamine 2000 (Invitrogen, Carlsbad, CA, USA), according to the manufactory's instruction.

\section{siRNAs and plasmids}

Extracted cDNA for BMPR2 and the MEG3 lncRNA were each cloned into the pcDNA 3.1 vector (Guangzhou Aiji Biotechnology Co., Ltd). Mimic miR-21, mimic NC, a miR-21 inhibitor, and a NC inhibitor were all purchased from the RuiBo Biotechnology Limited Company (Guangzhou, China). The BMPR2 or MEG3 IncRNA 3'-UTR fragment containing the predicted binding site for miR-21 or the corresponding mutant sequences were cloned into the pmirGLO Dual-Luciferase miRNA Target Expression Vector (Cat. \# E1330, Promega, Madison, WI, USA). siRNA fragments against BMPR2 (5' - GAGACUUAAGUGUUCAUUGAGAGGC 3'), MEG3 (5'-GGGCUUCUGGAAUGAGCAU-3'), or the scrambled control sequence (siNC, 5'-UUCUCCGAACGUGUCACGUTT-3'), which was designed to target no genes, were transfected into cells using Lipofectamine 2000 to knock down $B M P R 2$ or MEG3.

\section{Immunohistochemistry (IHC)}

The paraffin-embedded sections were heated in citrate buffer (pH 6.0) at $95^{\circ} \mathrm{C}$ for $15 \mathrm{~min}$ for antigen retrieval. The sections were then incubated in $3 \% \mathrm{H}_{2} \mathrm{O}_{2}$ for $20 \mathrm{~min}$ and were blocked using $10 \%$ goat serum (Sigma-Aldrich, Saint-Louis, USA) for $1 \mathrm{~h}$ to quench the endogenous peroxidase activity. They were then incubated with a primary antibody against BMPR2 (\#ab130206, 1:200, Abcam, Cambridge, MA, USA) for $1.5 \mathrm{~h}$ and with HRP-conjugated IgG (\#ab64264, 1:500, Abcam) for another $1 \mathrm{~h}$. The primary antibody was diluted in a QuickBlock buffer (\#P0262, Beyotime, Shanghai, China), and buffer without antibody was used as a negative control. The immunorections were developed using DAB reagent 
(Beyotime Institute of Biotechnology, Jiangsu, China) and were counterstained with hematoxylin (Beyotime). The tissue sections were analyzed and photographed with a microscope (BX60; Olympus, Tokyo, Japan).

\section{Quantitative reverse transcription PCR (RT-qPCR)}

Total RNA was extracted from tissues and cells using Trizol (Invitrogen, Carlsbad, CA, USA) and was reverse transcribed into cDNA using a reverse transcription kit (\#4368814, Invitrogen). The cDNA was amplified using the One-Step RT-PCR System Kit (Invitrogen) and SYBR Premix Ex Taq TM II (RR820A, TaKaRa Biotechnology, Japan). With U6 and GAPDH as controls, the relative transcription levels from each gene were calculated using the $2^{-\Delta \Delta C t}$ method. The primers targeting human BMPR2, MEG3, actin, and miR-21-5p were as follows: BMPR2-forward 5'-ACAGATAGGTGAGTCAACACAAGA-3' and BMPR2-reverse 5'TTGACTTCACAGTCCAGCGA-3'; $M E G 3$-forward 5'-GGCCTCCCCTTGAGTAGAGA-3' and MEG3-reverse 5'-ACTGAAGGCTCAACAGCTCC-3'; actin-forward 5'-CATGTACGTTGCTATCCAGGC-3' and actin-reverse 5'-CTCCTTAATGTCACGCACGAT-3'; miR-21-5p-forward 5'ACGGGTAGCTTATCAGACTGA-3' and miR-21-5p-reverse 5'CAGTGCGTGTCGTGGAGT-3'; miR-21-5p-RT, GTCGTATCCAGTGCGTGTC GTGGAGTCGGCAATTGCACTGGAT-
ACGACTCAACAT; and U6-forward 5'-CTCGCTTCGGCAGCACA-3' and U6-reverse 5'-AACGCTTCACGAATTTGCGT-3'.

\section{Western blotting}

Cells in the logarithmic growth phase were lysed with RIPA lysis buffer (\#P0013, Beyotime) containing protease and phosphatase inhibitors to extract proteins, and the BCA method was used to determine protein concentration. An equal amount of total protein $(50 \mu \mathrm{g})$ for each sample was loaded onto the $12 \%$ SDSPAGE gels used for electrophoresis, and the proteins were then transferred to a PVDF membrane (\#IPVH00010, Millipore, Burlington, MA, USA). The membrane was probed with primary antibodies against BMPR2 (\#ab130206, Abcam) and GAPDH (\#ab8245, Abcam) overnight at $4{ }^{\circ} \mathrm{C}$ followed by incubation with HRP-labeled secondary antibodies (Beyotime) at room temperature for $2 \mathrm{~h}$.

\section{CCK-8 assay}

Briefly, approximately $1 \times 10^{3}$ cells were inoculated onto a 96well plate in $100 \mu \mathrm{L}$ of fresh medium and were cultured at $37^{\circ} \mathrm{C}$ with $5 \% \mathrm{CO}_{2}$ for $0,24,48$, or $72 \mathrm{~h}$. Ten microliters of CCK-8 reagent (CA1210-100T, Solarbio, Beijing, China) was added to each well and incubated with the cells for $1 \mathrm{~h}$. The optical density at $450 \mathrm{~nm}$ was then assessed using a 96-well plate reader.
A

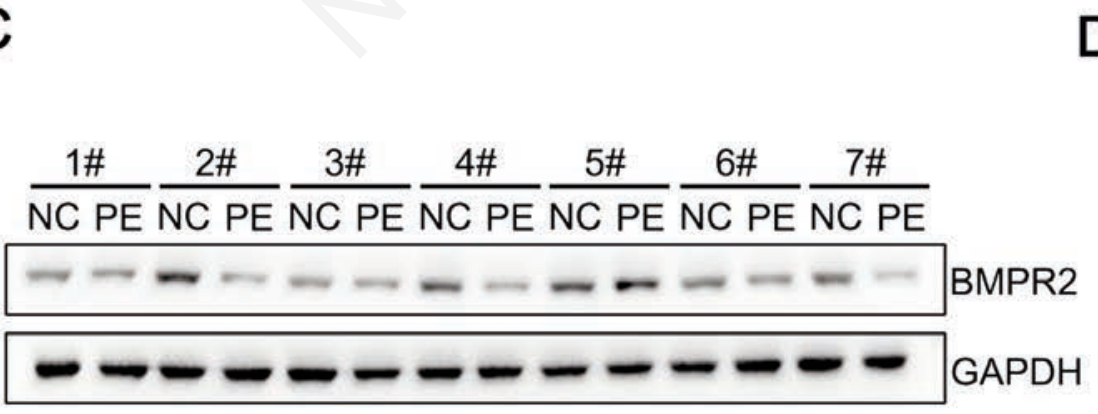

C

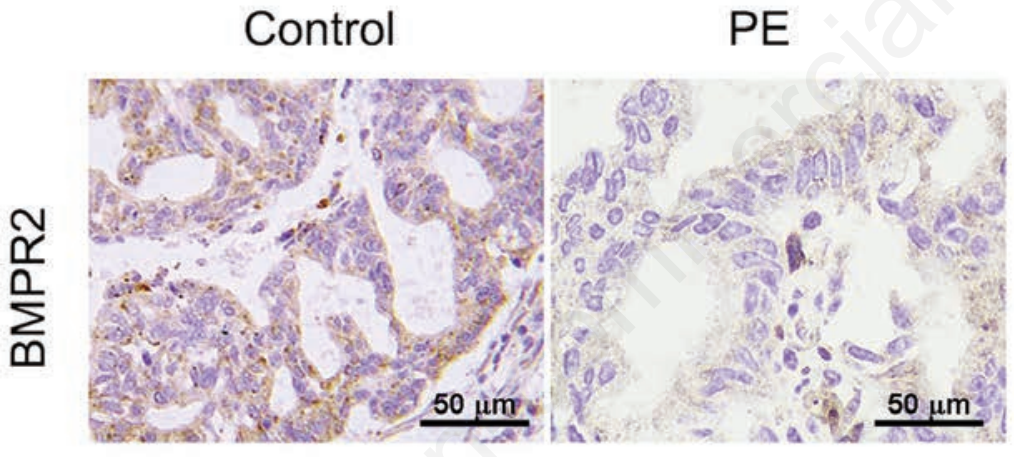

B
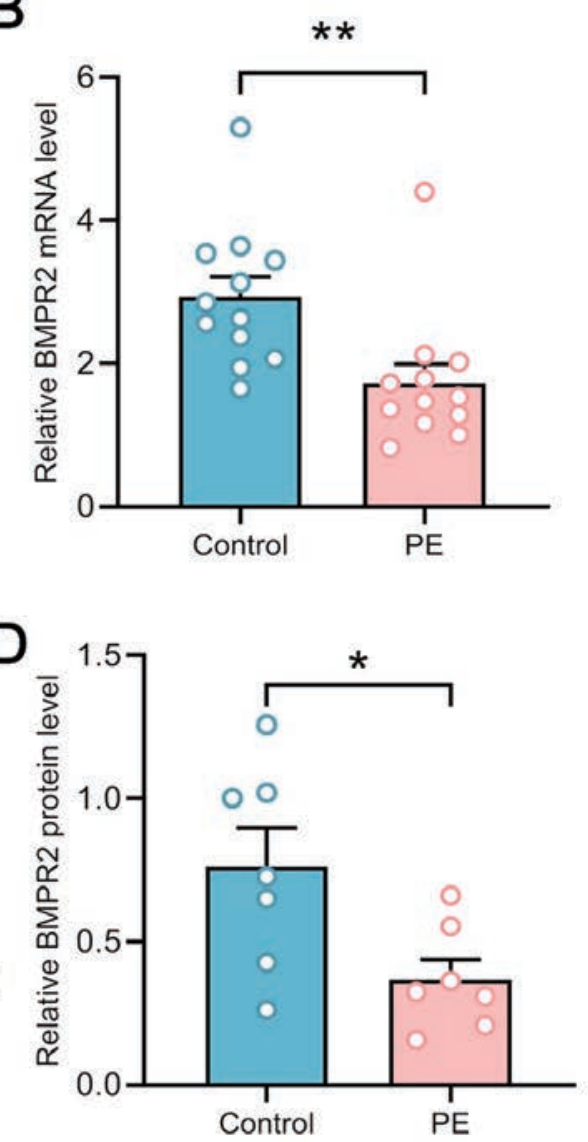

Figure 1. The expression level of BMPR2 was significantly downregulated in the PE placental tissues. A) Immunohistochemical staining for BMPR2 expression in the placental tissues for the normal pregnancy group and PE patients; 200x magnification; scale bar: 20 um. B) BMPR2 mRNA was detected in the normal pregnancy group and PE patients using RT-qPCR. C,D) Western blotting assays show the BMPR2 protein levels for the normal pregnancy group and PE patients; ${ }^{*} \mathbf{p}<0.05$ and ${ }^{* *} \mathrm{p}<0.01$ relative to the normal pregnancy group. Data are presented as the means $\pm S E M, n=3$. 


\section{Wound-healing assay}

Cells were plated on a 6 -well plate at a density of $5 \times 10^{5}$ cells/well and were cultured overnight until the cells adhered to the plate. A $200 \mu \mathrm{L}$ pipette tip was used to scrape the cell layer along the bottom of the 6-well plate, and the cells were photographed 0 and $24 \mathrm{~h}$ after scraping. For evaluation of wound closure, three randomly selected points along each wound were marked. The healing rate was expressed as percentage of scratch closure and was calculated as follows: $\%$ of scratch closure $=(a-b) / a$, where (a) is a distance between edges of the wound, and (b) is the distance which remained cell-free during cell migration to close the wound.

\section{Transwell invasion assay}

Transwell invasion assays were performed using transwell chambers (\#3422, Corning, NY, USA) to evaluate cell invasiveness. For the invasion assays, Transwell chambers were coated with $100 \mu 1$ Matrigel (BD Biosciences, Franklin Lakes, NJ, USA) and maintained at $37^{\circ} \mathrm{C}$ in a cell incubator with $5 \% \mathrm{CO}_{2}$. The JEG3 cells were resuspended in $200 \mu \mathrm{L}$ serum-free medium $\left(5 \times 10^{5}\right.$ cells $/ \mathrm{mL}$ ) and seeded in the upper chamber, and $600 \mu \mathrm{L}$ of medium containing $10 \%$ FBS was added to the lower chamber. After $24 \mathrm{~h}$ of incubation, the cells on the upper surface were removed with cotton swabs, and the lower layer was fixed with $4 \%$ paraformaldehyde and stained with $0.1 \%$ crystal violet (Beyotime). The number of invaded cells was measured using an
A

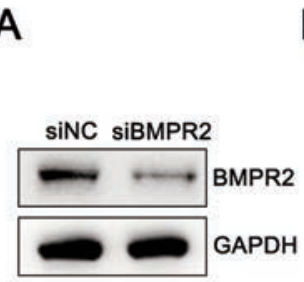

B

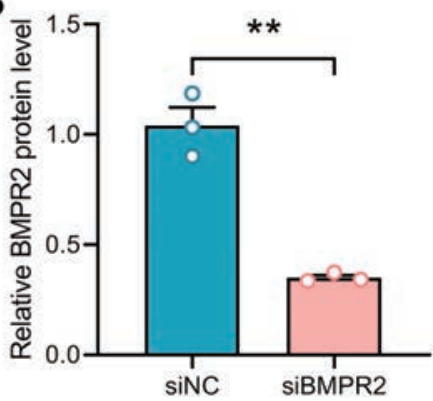

C

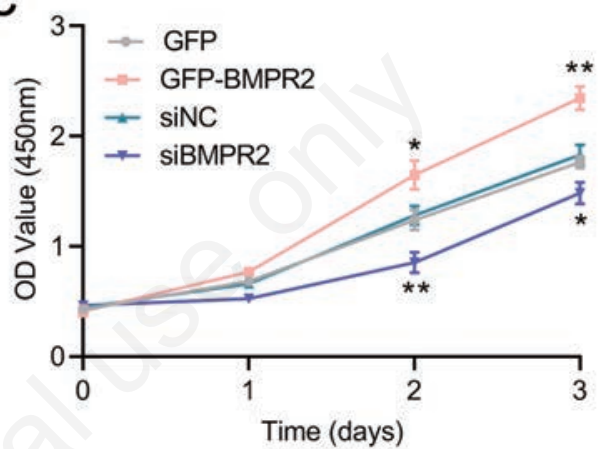

E

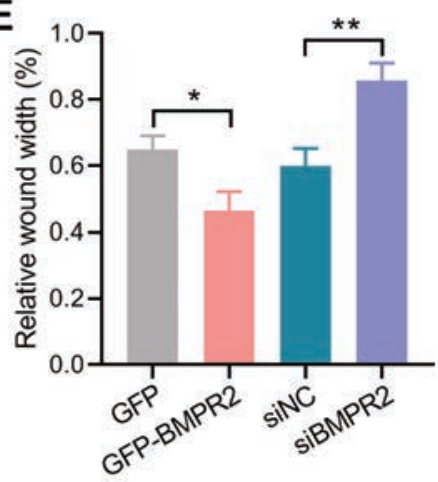

G

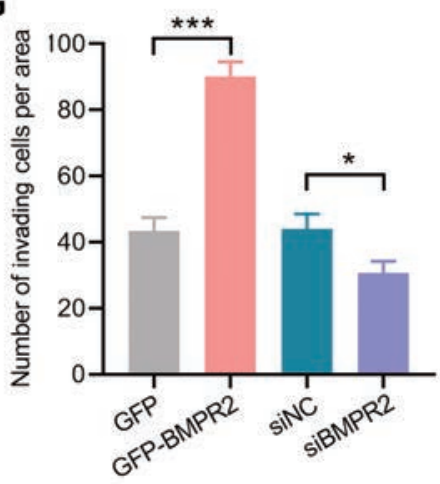

Figure 2. BMPR2 promotes JEG-3 cell proliferation and invasion. A,B) Western blotting analysis verifies the RNA interference efficiency for BMPR2 knockdown; ${ }^{* *} \mathrm{p}<0.01$ relative to the siNC group. C) CCK-8 assay for JEG-3 cell proliferation after treatment with siBMPR2, oe-BMPR2, or controls. D) Cell migration was measured using a wound-healing assay. E) Quantification of cell migration for JEG-3 cells treated with siBMPR2, oe-BMPR2, or controls. F) Invasiveness was analyzed using a transwell invasion assay. () Quantification of cell invasion; ${ }^{*} \mathrm{p}<0.05$ and ${ }^{* * *} \mathrm{p}<\mathbf{0 . 0 1}$ relative to the siNC or GFP group, respectively. Data are presented as the means \pm SEM, $n=3$. 
inverted microscope (200x magnification), from at least seven fields of each of three separate membranes.

\section{Dual-luciferase reporter gene assays}

JEG-3 cells were seeded onto a 24-well plate at a density of $5 \times 10^{4}$ cells/well and were incubated for $24 \mathrm{~h}$. The wild-type or mutant reporter genes were co-transfected with miR-21 or miRNC plasmids. Luciferase activity was determined using the DualLuciferase Reporter Assay System (Promega Corporation, Madison, WI, USA) $48 \mathrm{~h}$ after transfection.

\section{Statistical analysis}

Data were analyzed using the SPSS 19.0 software and are presented as the mean \pm SEM. All experiments were repeated at least three times. The statistical difference between two groups was determined using an independent $t$-test, and the Pearson correlation coefficient was used to analyze their correlation. $\mathrm{P}<0.05$ indicates a statistically significant difference.

\section{Results}

\section{BMPR2 is downregulated in the placenta tissues from PE patients}

To identify the BMPR2 expression level changes in PE, we collected placental tissues from PE and control pregnant women, and used IHC to detect protein levels. The results demonstrate that the expression level of BMPR2 was significantly reduced in the placenta tissue from PE patients (Figure 1A). RT-qPCR and western blotting analyses confirmed that the BMPR2 mRNA and protein levels, respectively, are reduced in the PE placenta tissues (Figure 1 B-D).

\section{BMPR2 increases the growth rate and invasiveness of JEG-3 cells}

To further explore the function of BMPR2, we performed in vitro experiments in the trophoblast cell line JEG-3, and transfected the cells with a plasmid encoding BMPR2 or siRNA fragments targeting BMPR2. The genetic knockdown efficiency was first confirmed using western blotting (Figure 1 A,B). Cell growth was

\section{A}

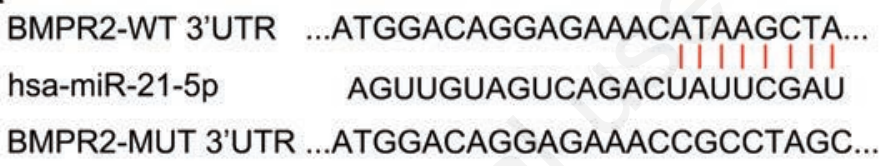

B

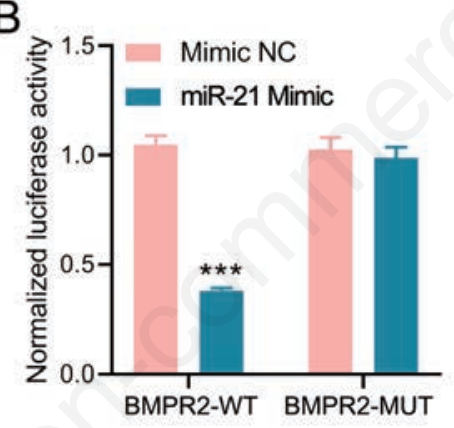

D

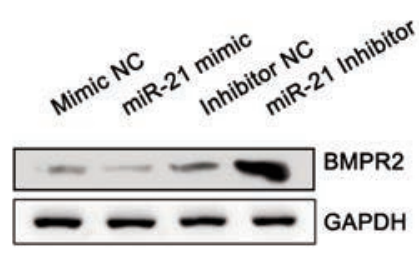

C

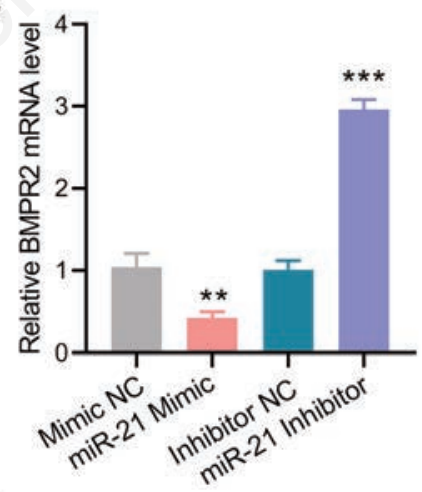

$\mathrm{E}$

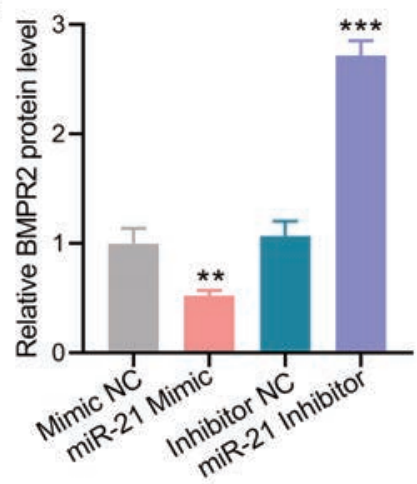

Figure 3. The targeting relationship between miR-21 and BMPR2. A) Bioinformatics analysis of the binding between $B M P R 2$ and miR21. B) Dual-luciferase reporter gene assays confirmed that miR-21 targets the $B M P R 2$ gene. C) RT-qPCR analysis of $B M P R 2$ mRNA transcription after treatment with a miR-21 inhibitor or a miR-21 mimic. $\mathrm{D}, \mathrm{E})$ Western blotting analysis of $B M P R 2$ protein expression after treatment with a miR-21 inhibitor or a miR-21 mimic; ${ }^{* *} \mathrm{p}<0.01$ and ${ }^{* *} \mathrm{p}<0.001$ relative to the mimic NC or inhibitor NC group, respectively. Data are presented as the means \pm SEM, $n=3$. 
determined using a CCK-8 assay. Overexpression of GFP-BMPR2 promoted JEG-3 cell growth, whereas $S i B M P R 2$ suppressed their growth (Figure 2C). As revealed by wound-healing and transwell assays, the levels of JEG-3 cell proliferation and invasion increased when BMPR2 was overexpressed in JEG-3 cells; knockdown of BMPR2 produced the opposite results (Figure 2 D-G). Collectively, these results indicate that BMPR2 promotes JEG-3 cell growth and invasiveness.

\section{miR-21 inhibits JEG-3 cell growth and invasiveness by} targeting BMPR2

To further identify the underlying mechanism of action of BMPR2 in PE, we performed bioinformatics analysis using the Targetscan $^{25}$ and starBase ${ }^{26}$ databases to screen for miRNAs that may bind to the 3'UTR of BMPR2 and found that miR-21-5p was most likely to regulate $B M P R 2$. To confirm this interaction, plasmids for wild-type (BMPR2-WT) and mutant BMPR2 (BMPR2MUT) with fluorescent signals were constructed (Figure $3 \mathrm{~A})$. For the dual-luciferase reporter gene assay, the miR-21 mimic and $B M P R 2-\mathrm{WT}$ - or BMPR2-MUT-encoding plasmids were co-trans-

A

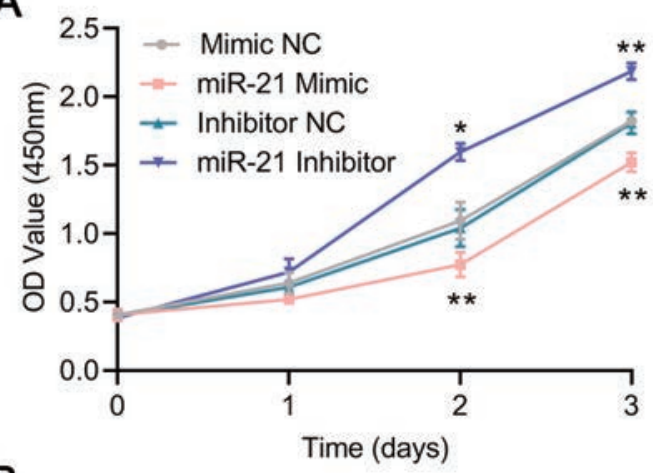

B

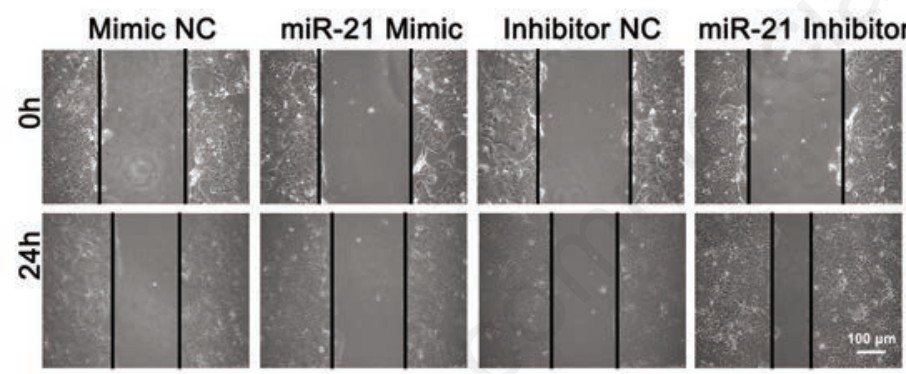

D

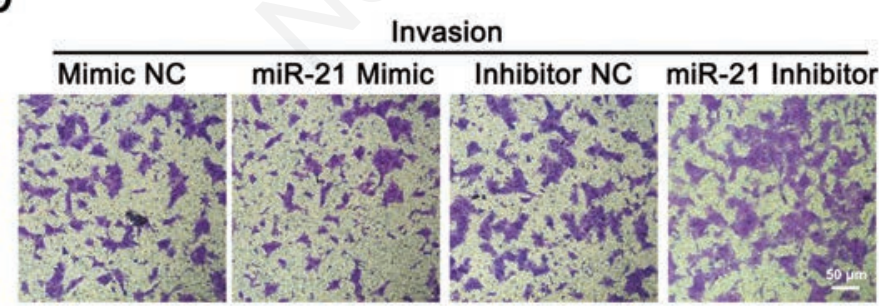

C

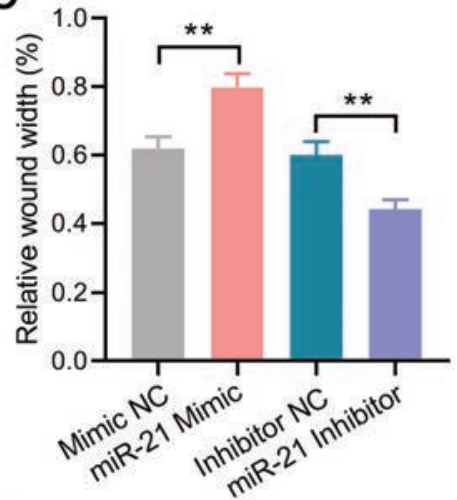

E

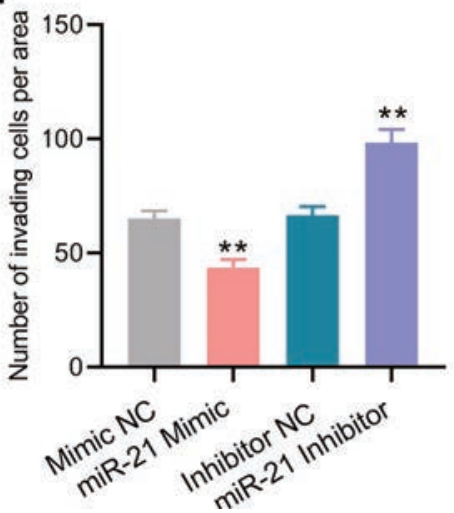

Figure 4. The inhibitory effect of miR-21 on JEG-3 cell proliferation and invasiveness. (A) A CCK-8 assay to measure JEG-3 cell proliferation after treatment with siBMPR2, oe-BMPR2, or controls. (B) Cell migration was measured using a wound-healing assay after treatment with a miR-21 inhibitor, a miR-21 mimic, or controls. (C) Quantification of JEG-3 cell migration. (D) Invasiveness was analyzed using a transwell invasion assay with cells treated with a miR-21 inhibitor, a miR-21 mimic, or controls. (E) Quantification of cell invasion. ${ }^{*} \mathrm{P}<0.05$ and ${ }^{*} \mathrm{P}<\mathbf{0 . 0 1}$ relative to the mimic $\mathrm{NC}$ or inhibitor $\mathrm{NC}$ group, respectively. Data are presented as the means \pm SEM, $n=4$. 
fected into JEG-3 cells, and the resultant luciferase activity was detected. The results show that co-transfection with the miR-21 mimic significantly decreased the observed luciferase activity with the $B M P R 2-W T$ plasmid, but no significant change was observed for BMPR2-MUT, indicating that miR-21 can bind to BMPR2 (Fig. $3 B$ ). In addition, transfection of the miR-21 mimic in JEG-3 cells decreased the mRNA transcription and protein expression levels of BMPR2, whereas transfection with the miR-21 inhibitor resulted in an increase in these levels (Figure $3 \mathrm{C}$-E). Furthermore, transfection with the miR-21 mimic decreased the proliferation of JEG3 cells and suppressed cell invasion; however, transfection with the miR-21 inhibitor produced the opposite effects and promoted cell growth and invasion (Figure $4 \mathrm{~A}-\mathrm{E}$ ). These results indicate that miR-21 targets BMPR2 and inhibits the expression of BMPR2, thereby suppressing JEG-3 cell growth and invasiveness.

$M E G 3$ promotes the growth and invasiveness of trophoblasts through the miR-21/BMPR2 signaling pathway

lncRNAs can sponge miRNAs and thereby inhibit their func- tion. Through bioinformatics analysis, we found that the lncRNA MEG3 might be the upstream regulator of miR-21 (Figure 5A). The results from RT-qPCR analysis in JEG-3 cells indicated that transfection of cells with the $M E G 3$ decreased the transcription level of miR-21, which was reversed by overexpression of the miR-21 mimic (Figure 5B). Genetic knockdown of MEG3 resulted in upregulation of miR-21, which was reversed by overexpressing the miR-21 inhibitor (Figure 5C). Furthermore, a dual-luciferase reporter gene assay showed that the miR-21 mimic suppressed the luciferase signal observed after transfection with the MEG3-WT plasmid, but this was not observed for the MEG3-MUT plasmid (Figure 5D). These data indicate that $M E G 3$ can sponge miR-21.

To verify the function of MEG3 in JEG-3 cells, siRNA fragments against $M E G 3$ were synthesized. Knockdown efficiency was confirmed using RT-qPCR (Fig. 6A). Cells were then transfected with siMEG3 fragments or a siNC control. CCK-8 and transwell assays were performed to evaluate the impact of these siRNAs on cell growth and invasiveness. Knockdown of the transcription level of $M E G 3$ decreased JEG-3 cell proliferation and invasiveness (Figure $6 \mathrm{~B}-\mathrm{F}$ ). MEG3 may regulate the transcription

A

MEG3-WT 3'UTR

hsa-miR-21-5p

MEG3-MUT 3'UTR

\section{5'-GGGGGCATTGGGCATTAAGCCCT-3' IIIII \\ 3'-AGUUGUAGUCAGACUAUUCGAU-5' \\ 5'-GGGGGCATTGGGCATGCGTACCT-3'}

B

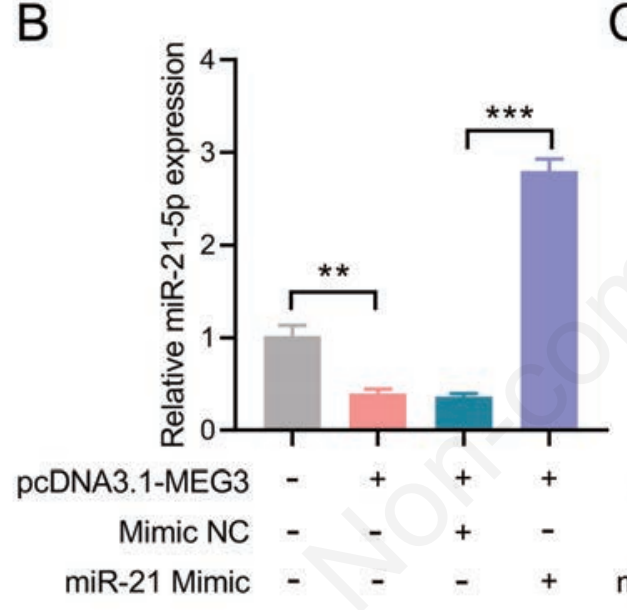

D

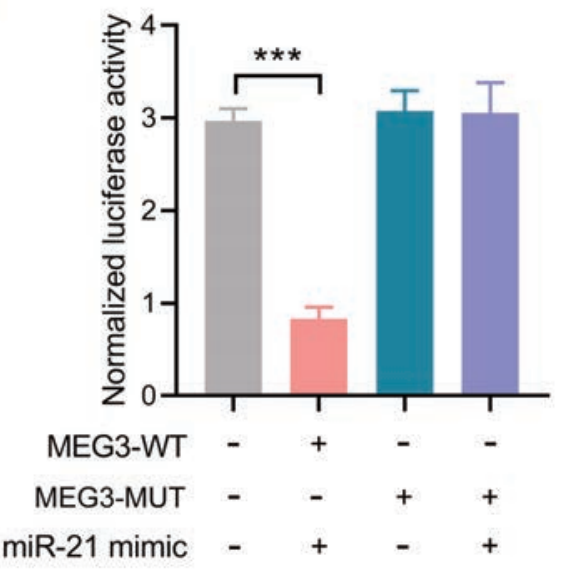

C

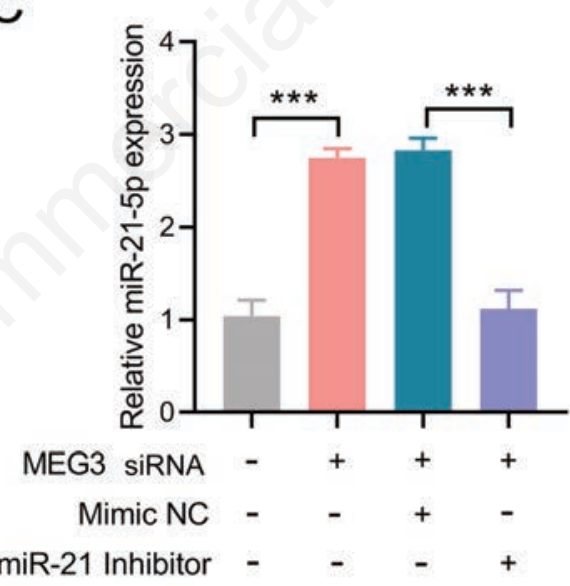

Figure 5. The targeting relationship between miR-21 and the IncRNA MEG3. A) Bioinformatics analysis showed that miR-21 is a potential target of the MEG3 IncRNA. B,C) RT-qPCR experiments verified that $M E G 3$ targets miR-21 and decreases its transcription level. D) A luciferase reporter gene experiment confirmed that MEG3 targets and regulates miR-21 transcription levels; ${ }^{* *} \mathbf{p}<0.01$ and ${ }^{* * *} \mathbf{p}<0.001$. Data are presented as the means \pm SEM, $\mathrm{n}=3$. 
level and function of miR-21 in cells. Therefore, we hypothesized that regulation of the transcription levels of these RNAs may play a role in PE. Using RT-qPCR, we investigated the levels of transcription in the placental tissues from the normal pregnancy group and PE patients, and found that miR-21 was upregulated (Figure $7 \mathrm{~A})$ and $M E G 3$ was downregulated in the PE tissues (Figure 7B). In addition, transcription of miR-21 was linearly negatively correlated with the transcription of MEG3 in PE tissues (Figure 7C).

Together, the above results indicate that, in vivo and in vitro, the lncRNA $M E G 3$ functions as an inhibitory factor that sponges miR-21, thereby regulating the transcription of $B M P R 2$ and promoting JEG-3 cell growth and invasiveness in PE.

A

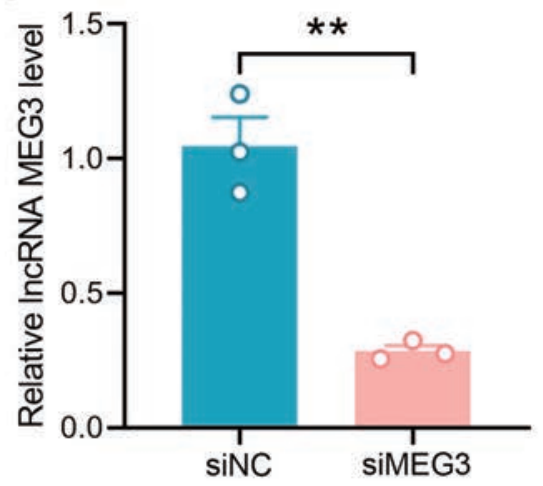

C

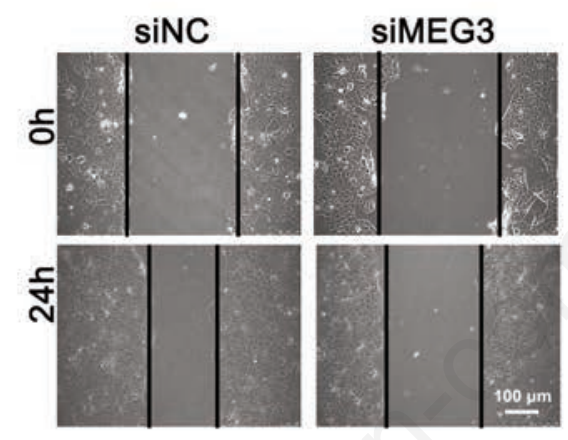

E

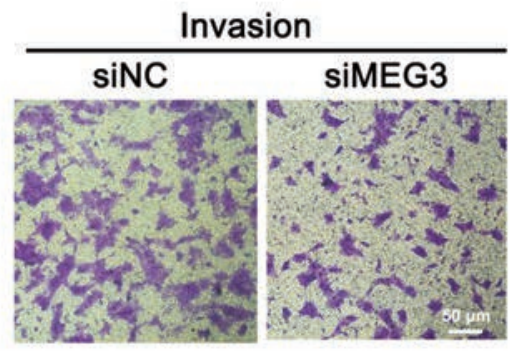

\section{Discussion}

Over the past few decades, extensive research from multiple angles has been conducted to explain the pathogenesis of PE and find effective treatment methods. Unfortunately, treatments including a $\mathrm{Na}^{+}$diet, $\mathrm{Ca}^{++}$supplementation, and diuretics have proven ineffective. ${ }^{27}$ Therefore, it is necessary to develop alternative options based on new understandings about the mechanisms of action underlying the development of PE.

TGF- $\beta$ family members play an important role in placenta formation and are also important pro-angiogenic factors. The level of
B

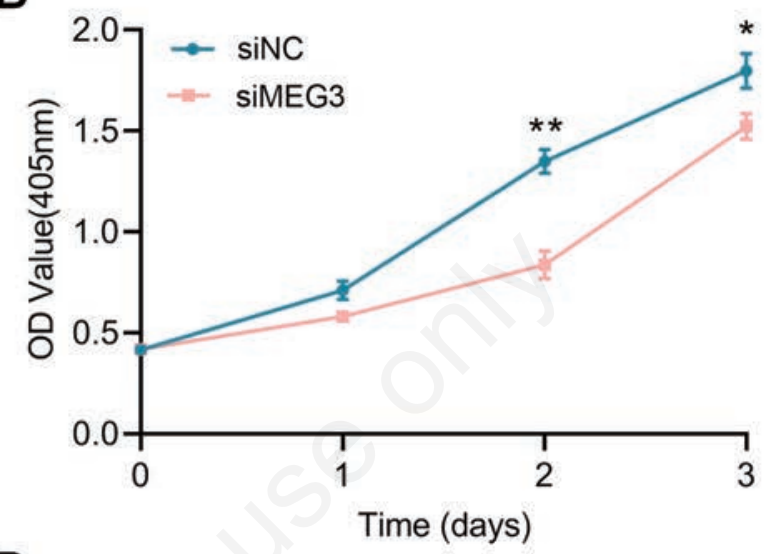

D

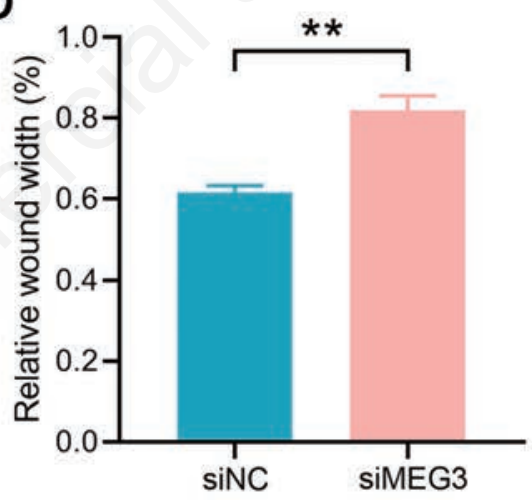

$\mathbf{F}$

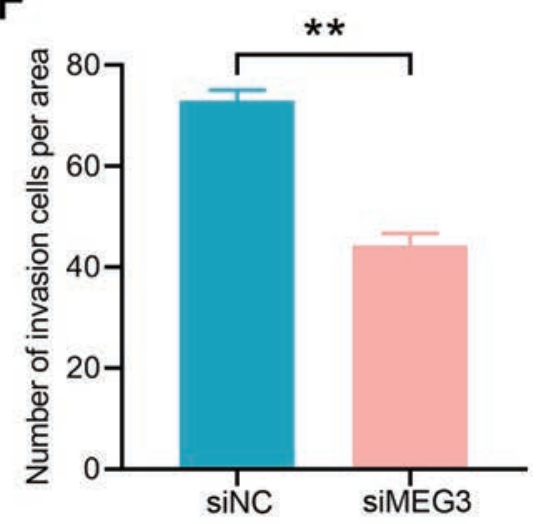

Figure 6. Impact of the lncRNA MEG3 on JEG-3 cell growth and invasiveness. A) A RT-qPCR experiment verified the interference efficiency of $s i M E G 3$. B) A CCK-8 assay for JEG-3 cell proliferation after treatment with siMEG3. C) Cell migration was measured using a wound-healing assay after treatment with siMEG. D) Quantification of JEG-3 cell migration. E) Invasiveness was analyzed using a transwell invasion assay with cells treated with siMEG3 or controls. F) Representative images from transwell assays for cells treated with siMEG3. Quantification of cell invasiveness is presented; ${ }^{* *} \mathrm{p}<0.01$ relative to the siNC group. Data are presented as the means $\pm S E M$, $\mathbf{n}=3$. 
soluble endoglin (sEng) is elevated in PE patient tissues. sEng is involved in neutralizing TGF- $\beta$, inhibiting the binding between TGF- $\beta$ and its TGF- $\beta$ receptor, and inducing vasodilation and endothelial nitric oxide synthase (eNOS) activation. ${ }^{28,29}$ BMP activates SMAD1/5/8 through BMP type I [activin A receptor type (ALK)1, 2, 3, and 6] and type II receptors. ${ }^{30}$ Our research focused on the role BMPR2 plays in PE. Previous studies investigated the function of BMPR2 in other vascular diseases, but little is known about its mechanism of action in PE. We found that BMPR2 is expressed at low levels in PE tissues, and overexpression of BMPR2 increased the growth and invasiveness of JEG-3 cells, indicating that BMPR2 plays an important role in the development of PE.

Because miRNAs have shown promise as biomarkers for various diseases, exploring the relationship between miRNAs and PE has become a topic of intense research interest. ${ }^{31}$ For example, miR-203a-3p is downregulated in the serum of PE patients, whereas overexpression of miRNA-203a-3p suppresses interleukin 24 (IL24) in THP-1 cells and inhibits inflammation. ${ }^{32}$ miR-125a-5p inhibits the migration and proliferation of trophoblast cells by targeting vascular endothelial growth factor A (VEGFA). ${ }^{33}$ miRNAs have the potential to become biomarkers for the diagnosis and treatment of PE, but further elucidation of the miRNA signaling network in PE is required. ${ }^{34-36}$ The observed levels of miR-21 transcribed in PE tissues are controversial. Relative to a normal pregnancy group, the level of transcription of miR-21 in late onset PE is low, but in early onset PE the level is not significantly different. ${ }^{37}$ These results are similar to those presented in an earlier miRNA analysis article. ${ }^{38}$ Most results suggest that the transcription of miR-21 increases in PE tissues. ${ }^{21,35}$ miR-21 targets FOXM1, thereby regulating the proliferation of placental cells and promoting the development of PE. ${ }^{39}$ In addition, the lncRNA Gas 5 regulates the PI3K/AKT signaling pathway and its downstream targets affect the biological functions of trophoblast cells, which are related to miR-21.40 Our results are in agreement with reports that observed high levels of transcription of miR-21 in PE. In addition, our results demonstrate that miR-21 inhibits JEG-3 growth and invasiveness by targeting BMPR2, providing some evidence and insight into understanding the miR-21 signaling network in PE.

In addition to miRNAs, IncRNAs are frequently involved in regulating the pathogenesis and development of PE. The lncRNA TCL6 is transcribed at high levels and promotes the development of PE by regulating transcription from CDK2 and PTEN. ${ }^{41}$ The lncRNA $S N H G 14$ acts as a molecular sponge for miR-330-5p to regulate trophoblast cell invasion and epithelial-mesenchymal transition (EMT). ${ }^{42}$ Knockdown of the transcription level of the lncRNA PVT1 reduces the expression of phosphorylated AKT and its downstream related genes, thereby inhibiting trophoblast cell growth. ${ }^{43}$ Herein, we show that $M E G 3$ acts as a molecular sponge of miR-21, which promotes the expression of BMPR2, and consequently, the function of JEG-3 cells. The function of $M E G 3$ in PE was preliminarily verified. $M E G 3$ affects the expression levels of

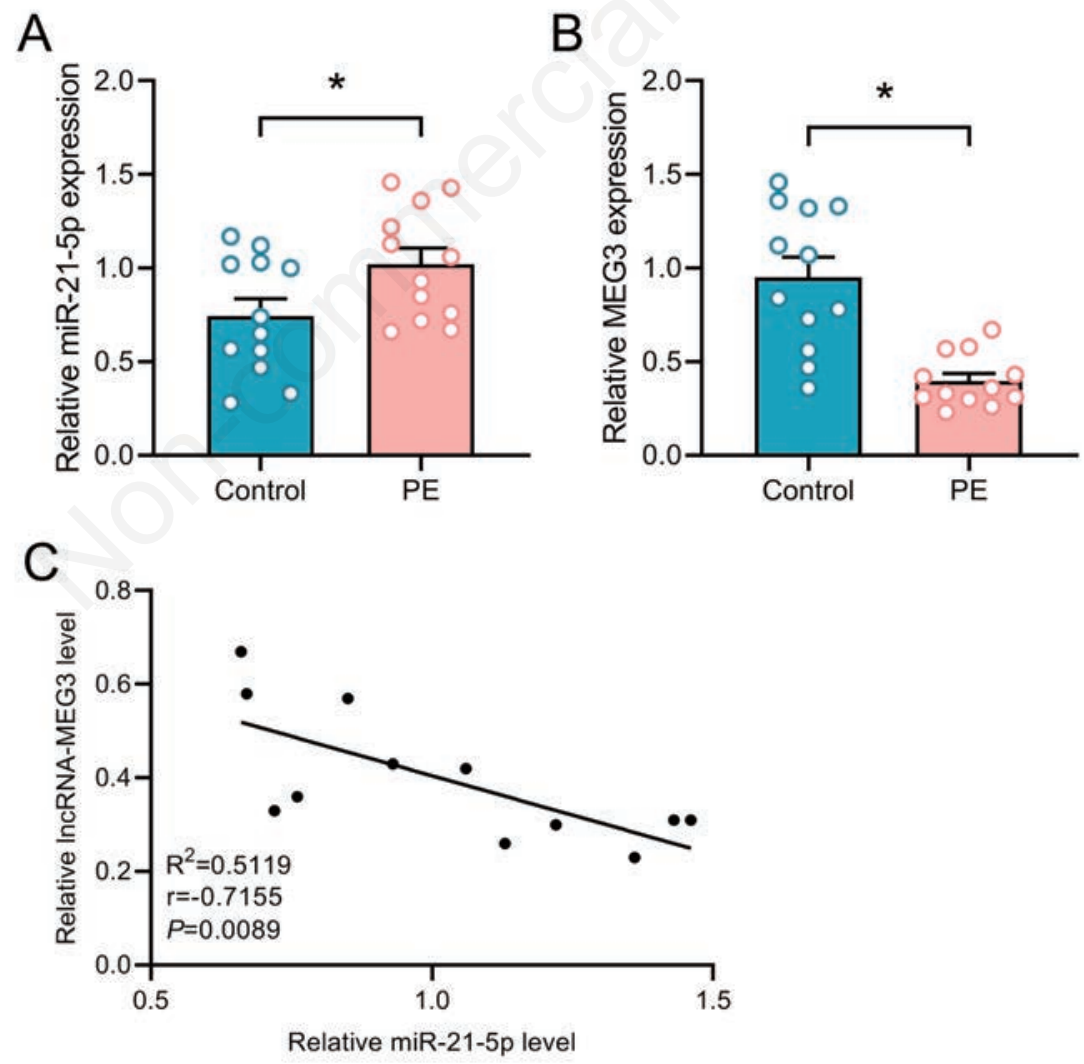

Figure 7. The IncRNA MEG3 promotes trophoblast cell metastasis and invasiveness through its regulation of the miR-21/BMPR2 signaling pathway. A,B) RT-qPCR analysis of the transcription levels of miR-21-5p and $M E G 3$ in the placenta for the normal pregnancy group and PE patients. C) Correlation analysis between the transcription levels of miR-21-5p and $M E G 3$ in the placental tissues of PE patients; ${ }^{*} \mathbf{p}<0.05$ relative to the normal pregnancy group. Data are presented as the means \pm SEM, $n=3$. 
nuclear factor kappa B $(\mathrm{NF}-\kappa \mathrm{B})$, Caspase-3, and Bax in trophoblast cells, thereby impacting cell apoptosis. ${ }^{44}$ In trophoblast cells, the upregulation of miR-21 inhibits $M E G 3$ transcription, thereby inhibiting EMT. ${ }^{45} M E G 3$ regulates the development of PE by targeting different signaling pathways, such as Ras-MAPK and Notch1.22,46 However, only one study showed that the MEG3/SMAD7 axis was involved in the development of PE through regulation of the TGF- $\beta$ signaling pathway and the EMT process in trophoblast cells. ${ }^{47}$ Therefore, this study gives us a deeper understanding of the relationship between MEG3 and the TGF$\beta$ signaling pathway.

In summary, this study shows that the lncRNA MEG3 increases trophoblast cell growth and invasiveness through the miR21/BMPR2 signaling pathway and inhibits the progress of PE. These results provide new insights into the pathogenesis of $\mathrm{PE}$ and will contribute to the development of novel treatments.

\section{References}

1. O'Callaghan KM, Kiely M. Systematic review of vitamin D and hypertensive disorders of pregnancy. Nutrients 2018;10:294.

2. Zhao X, Wang X. Candesartan targeting of angiotensin II type 1 receptor demonstrates benefits for hypertension in pregnancy via the NFkappaB signaling pathway. Mol Med Rep 2018;18:705-14.

3. Rhoads SJ, Serrano CI, Lynch CE, Ounpraseuth ST, Gauss CH, Payakachat N, et al. Exploring implementation of m-Health monitoring in postpartum women with hypertension. Telemed J E Health 2017;23:833-41.

4. Lim J, Cloete G, Dunsmuir DT, Payne BA, Scheffer C, von Dadelszen P, et al. Usability and feasibility of PIERS on the move: An mHealth app for pre-eclampsia triage. JMIR Mhealth Uhealth 2015;3:e37.

5. Lai WS, Ding YL. GNG7 silencing promotes the proliferation and differentiation of placental cytotrophoblasts in preeclampsia rats through activation of the mTOR signaling pathway. Int J Mol Med 2019;43:1939-50.

6. Salem MAA, Ammar IMM. First-trimester uterine artery pulsatility index and maternal serum PAPP-A and PlGF in prediction of preeclampsia in primigravida. J Obstet Gynaecol India 2018;68:192-6.

7. Shi Y, Massague J. Mechanisms of TGF-beta signaling from cell membrane to the nucleus. Cell 2003;113:685-700.

8. Sanchez-Duffhues G, Williams E, Goumans MJ, Heldin CH, Ten Dijke P. Bone morphogenetic protein receptors: Structure, function and targeting by selective small molecule kinase inhibitors. Bone 2020;138:115472.

9. Andruska A, Spiekerkoetter E. Consequences of BMPR2 deficiency in the pulmonary vasculature and beyond: Contributions to pulmonary arterial hypertension. Int J Mol Sci 2018;19:2499.

10. Luo L, Zheng W, Lian G, Chen $\mathrm{H}, \mathrm{Li} \mathrm{L}, \mathrm{Xu} \mathrm{C}$, et al. Combination treatment of adipose-derived stem cells and adiponectin attenuates pulmonary arterial hypertension in rats by inhibiting pulmonary arterial smooth muscle cell proliferation and regulating the AMPK/BMP/Smad pathway. Int J Mol Med 2018;41:51-60.

11. Dannewitz Prosseda S, Ali MK, Spiekerkoetter E. Novel advances in modifying BMPR2 signaling in PAH. Genes (Basel) 2020;12:8.

12. Wang J, Zhang C, Zhang Z, Zheng Z, Sun D, Yang Q, et al. A functional variant $\mathrm{rs} 6435156 \mathrm{C}>\mathrm{T}$ in BMPR2 is associated with increased risk of chronic obstructive pulmonary disease
(COPD) in Southern Chinese population. EBioMedicine 2016;5:167-74.

13. Ye F, Jiang W, Lin W, Wang Y, Chen H, Zou H, et al. A novel BMPR2 mutation in a patient with heritable pulmonary arterial hypertension and suspected hereditary hemorrhagic telangiectasia: A case report. Medicine (Baltimore) 2020;99:e21342.

14. Tanwar VS, Reddy MA, Natarajan R. Emerging role of long non-coding RNAs in diabetic vascular complications. Front Endocrinol (Lausanne) 2021;12:665811.

15. Zhang L, Xu X, Su X. Noncoding RNAs in cancer immunity: functions, regulatory mechanisms, and clinical application. Mol Cancer 2020;19:48.

16. Forero A, So L, Savan R. Re-evaluating strategies to define the immunoregulatory roles of miRNAs. Trends Immunol 2017;38:558-66.

17. Song P, Yang F, Jin H, Wang X. The regulation of protein translation and its implications for cancer. Signal Transduct Target Ther 2021;6:68.

18. Dragomir MP, Kopetz S, Ajani JA, Calin GA. Non-coding RNAs in GI cancers: from cancer hallmarks to clinical utility. Gut 2020;69:748-63.

19. Yan Y, Li XQ, Duan JL, Bao CJ, Cui YN, Su ZB, et al. Nanosized functional miRNA liposomes and application in the treatment of TNBC by silencing Slug gene. Int J Nanomedicine 2019;14:3645-67.

20. Saha S, Chakraborty S, Bhattacharya A, Biswas A, Ain R. MicroRNA regulation of transthyretin in trophoblast differentiation and intra-uterine growth restriction. Sci Rep 2017;7:16548.

21. Kolkova Z, Holubekova V, Grendar M, Nachajova M, Zubor P, Pribulova T, et al. Association of circulating miRNA expression with preeclampsia, its onset, and severity. Diagnostics (Basel) 2021;11:476.

22. Zhang J, Liu X, Gao Y. The long noncoding RNA MEG3 regulates Ras-MAPK pathway through RASA1 in trophoblast and is associated with unexplained recurrent spontaneous abortion. Mol Med 2021;27:70.

23. Yu Y, Wang L, Gao M, Guan H. Long non-coding RNA TUG1 regulates the migration and invasion of trophoblast-like cells through sponging miR-204-5p. Clin Exp Pharmacol Physiol 2019;46:380-8.

24. Wu HY, Wang XH, Liu K, Zhang JL. LncRNA MALAT1 regulates trophoblast cells migration and invasion via miR206/IGF-1 axis. Cell Cycle 2020;19:39-52.

25. Agarwal V, Bell GW, Nam JW, Bartel DP. Predicting effective microRNA target sites in mammalian mRNAs. Elife 2015;4:e05005.

26. Li JH, Liu S, Zhou H, Qu LH, Yang JH. starBase v2.0: decoding miRNA-ceRNA, miRNA-ncRNA and protein-RNA interaction networks from large-scale CLIP-Seq data. Nucleic Acids Res 2014;42:D92-7.

27. Jim B, Karumanchi SA. Preeclampsia: Pathogenesis, prevention, and long-term complications. Semin Nephrol 2017;37:386-97.

28. Venkatesha S, Toporsian M, Lam C, Hanai J, Mammoto T, Kim YM, et al. Soluble endoglin contributes to the pathogenesis of preeclampsia. Nat Med 2006;12:642-9.

29. Wedn AM, El-Bassossy HM, Eid AH, El-Mas MM. Modulation of preeclampsia by the cholinergic anti-inflammatory pathway: Therapeutic perspectives. Biochem Pharmacol 2021;192:114703.

30. Pera MF, Andrade J, Houssami S, Reubinoff B, Trounson A, Stanley EG, et al. Regulation of human embryonic stem cell differentiation by BMP-2 and its antagonist noggin. J Cell Sci 2004; 117:1269-80. 
31. Lv Y, Lu C, Ji X, Miao Z, Long W, Ding H, et al. Roles of microRNAs in preeclampsia. J Cell Physiol 2019;234:1052-61.

32. Ma HY, Cu W, Sun YH, Chen X. MiRNA-203a-3p inhibits inflammatory response in preeclampsia through regulating IL24. Eur Rev Med Pharmacol Sci 2020;24:5223-30.

33. Xueya Z, Yamei L, Sha C, Dan C, Hong S, Xingyu Y, et al. Exosomal encapsulation of miR-125a-5p inhibited trophoblast cell migration and proliferation by regulating the expression of VEGFA in preeclampsia. Biochem Biophys Res Commun 2020;525:646-53.

34. Hornakova A, Kolkova Z, Holubekova V, Loderer D, Lasabova Z, Biringer $\mathrm{K}$, et al. Diagnostic potential of MicroRNAs as biomarkers in the detection of preeclampsia. Genet Test Mol Biomarkers 2020;24:321-7.

35. Jairajpuri DS, Malalla ZH, Mahmood N, Almawi WY. Circulating microRNA expression as predictor of preeclampsia and its severity. Gene 2017;627:543-8.

36. Bounds KR, Chiasson VL, Pan LJ, Gupta S, Chatterjee P. MicroRNAs: New Players in the Pathobiology of Preeclampsia. Front Cardiovasc Med 2017;4:60.

37. Dong K, Zhang X, Ma L, Gao N, Tang H, Jian F, et al. Downregulations of circulating miR-31 and miR-21 are associated with preeclampsia. Pregnancy Hypertens 2019;17:59-63

38. Choi SY, Yun J, Lee OJ, Han HS, Yeo MK, Lee MA, et al. MicroRNA expression profiles in placenta with severe preeclampsia using a PNA-based microarray. Placenta 2013;34:799-804.

39. Zhou F, Sun Y, Gao Q, Wang H. microRNA-21 regulates the proliferation of placental cells via FOXM1 in preeclampsia. Exp Ther Med 2020;20:1871-8.
40. Zheng D, Hou Y, Li Y, Bian Y, Khan M, Li F, et al. Long noncoding RNA Gas5 is associated with preeclampsia and regulates biological behaviors of trophoblast via microRNA-21. Front Genet 2020;11:188.

41. Wu JL, Wang YG, Gao GM, Feng L, Guo N, Zhang CX. Overexpression of IncRNA TCL6 promotes preeclampsia progression by regulating PTEN. Eur Rev Med Pharmacol Sci 2019;23:4066-72.

42. Zhang Y, Zhang M. lncRNA SNHG14 involved in trophoblast cell proliferation, migration, invasion and epithelial-mesenchymal transition by targeting miR-330-5p in preeclampsia. Zygote 2021;29:108-17.

43. Wang Q, Lu X, Li C, Zhang W, Lv Y, Wang L, et al. Down-regulated long non-coding RNA PVT1 contributes to gestational diabetes mellitus and preeclampsia via regulation of human trophoblast cells. Biomed Pharmacother 2019;120:109501.

44. Zhang Y, Zou Y, Wang W, Zuo Q, Jiang Z, Sun M, et al. Downregulated long non-coding RNA MEG3 and its effect on promoting apoptosis and suppressing migration of trophoblast cells. J Cell Biochem 2015;116:542-50.

45. Wang R, Zou L, Yang X. microRNA-210/ Long non-coding RNA MEG3 axis inhibits trophoblast cell migration and invasion by suppressing EMT process. Placenta 2021;109:64-71.

46. Wang R, Zou L. Downregulation of LncRNA-MEG3 promotes HTR8/SVneo cells apoptosis and attenuates its migration by repressing Notch1 signal in preeclampsia. Reproduction 2020;160:21-9.

47. Yu L, Kuang LY, He F, Du LL, Li QL, Sun W, et al. The role and molecular mechanism of long nocoding RNA-MEG3 in the pathogenesis of preeclampsia. Reprod Sci 2018;25:1619-28.

Received for publication: 6 September 2021. Accepted for publication: 19 October 2021.

This work is licensed under a Creative Commons Attribution-NonCommercial 4.0 International License (CC BY-NC 4.0).

(C) Copyright: the Author(s), 2021

Licensee PAGEPress, Italy

European Journal of Histochemistry 2021; 65:3323

doi:10.4081/ejh.2021.3323

OPEN 